\title{
Imaging of the 6-OPRI Mutation Prion Disease: An Entity Distinct from Typical Creutzfeldt-Jakob Disease?
}

I $\mathrm{n}$ the article "Multi-parameter MR Imaging in the 6-OPRI Variant of Inherited Prion Disease," featured in this issue, De Vita et $\mathrm{al}^{1}$ evaluate a particular mutation of human prion disease, thereby making an essential contribution to our understanding of the clinical and radiologic spectrum of this disorder. The surprising results suggest that the 6-octapeptide repeat insertion (6-OPRI) mutation does not demonstrate the typical imaging findings of some genetic variants of Creutzfeldt-Jakob disease (CJD), such as the E200K variant, which Prohovnik and colleagues established as clinically and radiographically equivalent to sporadic CJD in a study using high-b-value diffusion-weighted imaging and which other authors illustrated with the V210I mutation. ${ }^{2,3}$ Because it is not yet known whether this finding applies to other mutations, the study of De Vita et al may help in further understanding the diversity of familial CJD populations.

The group of individuals studied was found to have an inherited prion disease related to a 6-OPRI mutation in a cohort of families in the United Kingdom. Although the sample size was small, no other group has access to such a substantial number of cases with this particular mutation. The present study aimed to explore underinvestigated aspects of cerebral degeneration in this condition, namely systematic voxel-based morphometry, magnetization transfer, and more standard diffusivity methods.

When one reviews this article, it seems that more questions are raised surrounding the nature of this particular cohort of familial CJD. These findings and, more important, the lack of findings characteristic of sporadic CJD in other inherited prion diseases suggest that the 6-OPRI is distinctive, with divergent clinical and imaging findings. Could this constitute a great enough difference to be classified independent of other familial forms of CJD? Do these divergent imaging findings explain the prominent early cognitive and neurodevelopmental-pattern deficits in this specific mutation, which make it different from other familial prion diseases? CJD and other associated prion diseases related to the abnormal conformation of a normal protein found in the brain, though uncommon, feature prominently in the differential diagnosis in patients with presenile dementia. Sporadic, familial, iatrogenic, and variant (or new variant) forms of CJD exist. An extensive body of neuroimaging research during the past 2 decades rapidly established the importance of MR imaging, particularly diffusion-weighted imaging, in solidifying the diagnosis of CJD. ${ }^{4}$ Multiple forms of familial CJD exist, with associated genetic mutations, and constitute a substantial minority of cases. The presence of autosomal dominant inherited mutations in cohorts of families with CJD offers the unique opportunity to model progression of imaging changes before symptoms.

Previous imaging evidence of this particular mutation demonstrated cortical thinning within predominantly the posterior frontal and parietal lobes. ${ }^{5}$ This present study extended the findings of this previous work in using voxel-based analysis of advanced MR imaging in several patients with 6-OPRI mutations. The authors discovered volume reductions, decreases in magnetization transfer ratio, and diffusivity increases within subcortical gray matter structures as well as some cortical structures including the perisylvian cortex and precuneus.

There are some clinical differences between patients with 6-OPRI and those with other prion-related mutations such as $P 102-L$ that may explain the divergent imaging profile. Patients with this particular mutation have lower premorbid cognitive functioning, which may predate the neurodegenerative changes. It is perhaps for this reason that cortical thinning and now the notable volume reduction and increased diffusivity in the cortical and subcortical gray matter structures feature prominently in this particular mutation, despite the absence of more typical morphologic imaging findings. This study notably differs from the imaging findings seen in other reported forms of familial CJD, including the $E 200 \mathrm{~K}$ mutation and V210I, both of which are thought to resemble sporadic CJD with prototypical restricted diffusion in the basal ganglia and thalamus. ${ }^{3}$ Although there are substantial similarities among sporadic CJD, variant CJD, and several genetic variants on MR imaging, ${ }^{3}$ it is surprising that the 6-OPRI mutation appears to be very different from all other variants.

The authors of this work as well as others in the field of prion disease imaging benefitted greatly from the work of our late colleague, Isak Prohovnik, who, before his passing, assisted greatly in critiquing and amending the work of De Vita et al. ${ }^{1}$ Dr Prohovnik's own research, particularly in establishing an extensive prospective imaging cohort of patients with the E200K mutation, 
advanced the field of CJD imaging tremendously. In building on the pioneering work of our esteemed colleague, Isak Prohovnik, and applying his multidisciplinary approach combining clinical and imaging resources to the study of 6-OPRI, the authors of this study further advance our understanding of the diversity of prion diseases.

\section{REFERENCES}

1. De Vita E, Ridgway G, Scahill R, et al. Multi-parameter MRI in the 6-OPRI variant of inherited prion disease. AJNR Am J Neuroradiol 2013;34:1723-30

2. Lee H, Hoffman C, Kingsley PB, et al. Enhanced detection of diffusion reductions in Creutzfeldt-Jakob disease at a higher $\mathbf{B}$ factor. AJNR Am J Neuroradiol 2010;31:49-54

3. Breithaupt M, Romero C, Kallenberg K, et al. Magnetic resonance imaging in E200K and V210I mutations of the prion protein gene. Alzheimer Dis Assoc Disord 2013;27:87-90

4. Zerr I, Kallenberg K, Summers DM, et al. Updated clinical diagnostic criteria for sporadic Creutzfeldt-Jakob disease. Brain 2009;132: 2659-68

5. Alner K, Hyare H, Mead S, et al. Distinct neuropsychological profiles correspond to distribution of cortical thinning in inherited prion disease caused by insertional mutation. J Neurol Neurosurg Psychiatry 2012;83:109-14

A.J. Degnan

L.M. Levy

http://dx.doi.org/10.3174/ajnr.A3490 